\title{
Bronchopulmonary dysplasia (BPD) rates in preterm infants - is there a change with non-invasive ventilation?
}

\author{
Dr Magdalena Fuller, ST6; Dr Claire Slater, ST7 and Dr Vennila Ponnusamy, Consultant \\ Neonatologist, Neonatal Intensive Care Unit, St Peter's Hospital, Chertsey, UK
}

\section{Background}

- Incidence of BPD remains around $40 \%$ in developed countries using newer definition of need for supplemental oxygen or pressure at 36 weeks post menstrual age ${ }^{1}$

- $\quad$ Further research suggests more reliable cut off for definition as 40 weeks post menstrual age There is increasing use of non-invasive ventilation reducing trauma to the developing lungs We wanted to compare our unit's data against UK National Neonatal Audit Programme (UK NNAP)

\section{Methods}

Review of neonates born between January to December 2016, <32 weeks gestation, admitted to NICU at SPH

Data collected via Evolve electronic medical records and BadgerNet database

Babies categorised into 4 groups based on gestation and place of birth
- Group 1: born at SPH $<30$ weeks
- Group 2: born elsewhere <30 weeks
- Group 3: born at SPH 30-32 weeks
- Group 4: born elsewhere 30-32 weeks

- Babies were excluded if they died, transferred in for special care or other short stay in the unit

\section{Results}

105 eligible babies were identified

47 babies $<30$ weeks were born at SPH and 35 babies $<30$ weeks were born elsewhere and transferred in

19 babies at 30-32 weeks were born at SPH and 4 were born elsewhere and transferred in

Table 1 shows baseline clinical characteristics.

\begin{tabular}{|l|l|l|l|l|}
\hline (Mean) & Group 1 & Group 2 & Group 3 & Group 4 \\
\hline $\begin{array}{l}\text { Gestation } \\
\text { (weeks) }\end{array}$ & $27+4$ & $27+3$ & $30+2$ & $30+4$ \\
\hline $\begin{array}{l}\text { Birthweight } \\
\text { 960g }\end{array}$ & $1040 \mathrm{~g}$ & $1567 \mathrm{~g}$ & $1340 \mathrm{~g}$ \\
\hline $\begin{array}{l}\text { Ventilation } \\
\text { BiPAP + CPAP } \\
\text { (days) }\end{array}$ & $1 \mathrm{~d}$ & $5 \mathrm{~d}$ & $0 \mathrm{~d}$ & $3 \mathrm{~d}$ \\
\hline $\begin{array}{l}\text { Vapotherm } \\
\text { (days) }\end{array}$ & $25 \mathrm{~d}$ & $22 \mathrm{~d}$ & $4 \mathrm{~d}$ & $3.5 \mathrm{~d}$ \\
\hline $\begin{array}{l}\text { Nasal cannula } \\
\text { oxygen (days) }\end{array}$ & $22 \mathrm{~d}$ & $16 \mathrm{~d}$ & $0 \mathrm{~d}$ & $2.5 \mathrm{~d}$ \\
\hline
\end{tabular}

Table 1. Baseline clinical characteristics according to groups

Table 2 shows risk factors for BPD and treatments received while inpatient

Overall, our rate of BPD at $47.6 \%$ is higher than UK national average for level 3 NICUs $(38 \%)^{2}$

With increasing gestation we show that the rate of BPD falls. (Table 3)
Babies in Group 4 had different pathology requiring oxygen or ventilation, not BPD. This group was also small with only 4 babies included, reflecting higher percentages.

\begin{tabular}{|c|c|c|c|c|c|}
\hline & Group 1 & Group 2 & Group 3 & Group 4 & Overall \\
\hline $\begin{array}{l}\text { Antenatal } \\
\text { steroids }\end{array}$ & $\begin{array}{l}93.6 \% \\
(44 / 47)\end{array}$ & $\begin{array}{l}91.4 \% \\
(32 / 35)\end{array}$ & $\begin{array}{l}100 \% \\
(19 / 19)\end{array}$ & $\begin{array}{l}75 \% \\
(3 / 4)\end{array}$ & $\begin{array}{l}95.2 \% \\
(98 / 105)\end{array}$ \\
\hline $\begin{array}{l}\text { Complete } \\
\text { course of } \\
\text { steroids } \\
\end{array}$ & $\begin{array}{l}72.3 \% \\
(34 / 47)\end{array}$ & $\begin{array}{l}54.3 \% \\
(19 / 35)\end{array}$ & $\begin{array}{l}100 \% \\
(19 / 19)\end{array}$ & $\begin{array}{l}50 \% \\
(2 / 4)\end{array}$ & $\begin{array}{l}72.4 \% \\
(74 / 105)\end{array}$ \\
\hline Infection risk & $\begin{array}{l}85.1 \% \\
(40 / 47)\end{array}$ & $\begin{array}{l}91.4 \% \\
(32 / 35)\end{array}$ & $\begin{array}{l}78.9 \% \\
(15 / 19)\end{array}$ & $\begin{array}{l}100 \% \\
(4 / 4)\end{array}$ & $\begin{array}{l}86.7 \% \\
(91 / 105)\end{array}$ \\
\hline $\begin{array}{l}\text { PPROM >7 } \\
\text { days }\end{array}$ & $\begin{array}{l}29.8 \% \\
(14 / 47)\end{array}$ & $\begin{array}{l}37.1 \% \\
(13 / 35)\end{array}$ & $\begin{array}{l}36.8 \% \\
(7 / 19)\end{array}$ & $\begin{array}{l}25 \% \\
(1 / 4)\end{array}$ & $\begin{array}{l}33.3 \% \\
(35 / 105)\end{array}$ \\
\hline Birth by LSCS & $\begin{array}{l}70.2 \% \\
(33 / 47)\end{array}$ & $\begin{array}{l}60 \% \\
(22 / 35)\end{array}$ & $\begin{array}{l}57.9 \% \\
(11 / 19)\end{array}$ & $\begin{array}{l}75 \% \\
(3 / 4)\end{array}$ & $\begin{array}{l}64.8 \% \\
(68 / 105)\end{array}$ \\
\hline Surfactant & $51.1 \%$ & $85.7 \%$ & $31.6 \%$ & $100 \%$ & $61 \%$ \\
\hline $\begin{array}{l}\text { Surfactant } \\
\text { timing } \\
\text { (median) } \\
\end{array}$ & $\begin{array}{l}0.5 \mathrm{~h} \text { from } \\
\text { birth }\end{array}$ & $\begin{array}{l}\text { Oh from } \\
\text { birth }\end{array}$ & $\begin{array}{l}11 \mathrm{~h} \\
\text { from } \\
\text { birth }\end{array}$ & $\begin{array}{l}\text { 6h from } \\
\text { birth }\end{array}$ & $\begin{array}{l}\text { Oh from } \\
\text { birth }\end{array}$ \\
\hline $\begin{array}{l}\text { Postnatal } \\
\text { steroids }\end{array}$ & $25.5 \%$ & $25.7 \%$ & $10.5 \%$ & $0 \%$ & $21.9 \%$ \\
\hline Home oxygen & $38.3 \%$ & $42.9 \%$ & $10.5 \%$ & $25 \%$ & $35.2 \%$ \\
\hline & \multicolumn{2}{|l|}{28 days } & 36 weeks & \multicolumn{2}{|c|}{40 weeks } \\
\hline Group 1 & \multicolumn{2}{|l|}{$79 \%$} & $62 \%$ & \multicolumn{2}{|l|}{$43 \%$} \\
\hline Group 2 & \multicolumn{2}{|l|}{$63 \%$} & $49 \%$ & \multicolumn{2}{|l|}{$46 \%$} \\
\hline Group 3 & \multicolumn{2}{|l|}{$16 \%$} & $16 \%$ & \multicolumn{2}{|l|}{$16 \%$} \\
\hline Group 4 & \multicolumn{2}{|l|}{$50 \%$} & $50 \%$ & \multicolumn{2}{|l|}{$25 \%$} \\
\hline Overall & \multicolumn{3}{|l|}{$60 \%$} & \multicolumn{2}{|l|}{$37 \%$} \\
\hline
\end{tabular}

Table 3. Percentage of BPD rates according to different definitions

\section{Conclusion}

Our rates of BPD are high despite non-invasive management Review of practice is necessary to improve outcomes. This includes earlier and effective use of surfactant, as well as streamlining treatment with postnatal steroids, and improvement in nutrition in at risk infants.

Closer attention needs to be paid to babies nearing 36 weeks post menstrual age and review whether oxygen therapy is clinically indicated and why

Using cut off of 40 weeks post menstrual age might prove to be more clinically relevant

\section{References}

1. Stoll B.J., Hansen N.I., Bell E.F., Shankaran S., Laptook A.R., Walsh M.C., Hale E.C., Newman N.S., Schibler K., Carlo W.A., et al. Neonatal outcomes of extremely preterm infants from the nichd neonatal research network. Pediatrics. 2010;126:443-456. doi: 10.1542/peds.2009-2959

2. http://www.nnap.rcpch.ac.uk/annual-reports.aspx 